\title{
VIEWPOINT
}

\section{Die and let live: harnessing BikDD to combat breast cancer stem cells}

\author{
Mario Giuliano', Meghana V Trivedi’,2 and Rachel Schiff $1,3,4,5, *$
}

\begin{abstract}
One of the possible mechanisms contributing to the intrinsic resistance of cancer stem cells (CSCs) to conventional therapies is the inefficiency of activating the apoptotic machinery. In a recent study by Lang and colleagues, the engineered constitutively active pro-apoptotic protein BikDD, which works by inhibiting multiple Bcl-2 family members, was tested in various preclinical breast cancer models. Delivered to cells via an innovative cancer cell-specific gene-therapy approach, BikDD showed potent activity against CSCs and synergized with lapatinib and paclitaxel treatment. This novel and promising therapy warrants further translation to the clinic
\end{abstract}

\section{Background}

Despite significant improvements in breast cancer therapy achieved in the last decades, tumor progression occurs often and is associated with high mortality. Treatment resistance and disease progression are thought to occur due to the inability of conventional therapies to eradicate all the tumor cells, especially the cancer stem cells (CSCs) [1]. These CSCs are resistant to chemotherapy and radiation, capable of self-renewal, and thus responsible for tumor progression $[1,2]$. No drugs are currently available in the clinic to effectively and selectively target CSCs.

One of the potential mechanisms of CSC-related treatment resistance is their unique survival property, which enables them to escape from drug-induced apoptosis [3]. It has been shown in both hematologic and solid tumors that CSCs express high levels of mRNAs encoding antiapoptotic proteins, including $\mathrm{Bcl}-2$ family members $[4,5]$. In addition, overexpression of $\mathrm{Bcl}-2$ and its family members has been associated with resistance to various chemotherapeutic drugs as well as targeted therapies, in

*Correspondence: rschiff@bcm.edu

'Lester and Sue Smith Breast Center at Baylor College of Medicine, Houston,

TX 77030, USA

Full list of author information is available at the end of the article both preclinical and clinical settings [6-9]. Because the anti-apoptotic machinery can be regulated by several mechanisms in CSCs, this redundancy presents a challenging obstacle for the development of effective proapoptotic treatments [10].

\section{The article}

In line with these premises, Lang and colleagues [11] studied the anti-tumor activity of BikDD, a constitutively active form of the pro-apoptotic protein Bik. The authors found that BikDD effectively targeted the breast CSCs by inhibiting multiple anti-apoptotic $\mathrm{Bcl}-2$ family members (Bcl-2, Bcl-xL, Bcl-W, and Mcl-1), significantly reducing the percentage of $\mathrm{CD} 44^{+} / \mathrm{CD} 24-\mathrm{CSCs}$, and impairing mammosphere formation in various pre-clinical models of breast cancer. BikDD also reduced tumor-initiating capacity of mammospheres injected into NOD/SCID mice. Importantly, while silencing of individual $\mathrm{Bcl}-2$ family members by short hairpin RNA (shRNA) knock down had only a modest effect on the survival of CSCs, co-silencing by either combined shRNAs or BikDD resulted in significant CSC reduction.

Additionally, the authors integrated the BikDD gene in a vector-liposome complex named VISA-claudin4BikDD for selective delivery to and expression in breast cancer cells. The complex inhibited tumor cell growth in vitro and in vivo, with only limited effects on normal cells and low toxicity in mice. Inadequate apoptosis can limit the efficacy of HER2-targeting drugs such as lapatinib $[8,9]$, and BikDD gene therapy enhanced lapatinib efficacy in HER2-positive and epidermal growth factor receptor (EGFR)-positive breast cancer models in vitro and in vivo, though no complete tumor regressions were observed. Lastly, the combination of VISA-claudin4BikDD with the chemotherapeutic drug paclitaxel prevented the CSC increase observed after paclitaxel alone and significantly reduced tumor growth in vivo, even after the cessation of treatment. Thus, BikDD may also target chemotherapy-resistant CSCs.

\section{The viewpoint}

Several lines of evidence emphasize the importance of targeting CSCs to improve treatment outcome in breast 
cancer. Nevertheless, only a few preclinical studies in this effort have shown positive results to date [3,12]. Resistance of CSCs to drug-induced apoptosis [10] suggests that approaches to induce apoptosis in CSC populations would be promising. Furthermore, apoptosis-inducing treatments, such as tumor necrosis factor-related apoptosis inducing ligand (TRAIL) agonists and Bcl-2 antagonists, have yielded positive results in the preclinical setting and are already under clinical investigation in breast cancer and other malignancies [13,14]. However, only a limited number of studies have explored the direct role of pro-apoptotic treatments in CSCs [15].

Lang and colleagues have conducted a pioneering study of the novel and potent pro-apoptotic molecule BikDD, which was used to target breast CSCs. The innovation of their approach is two-fold. First, BikDD can inhibit all the major Bcl-2 anti-apoptotic family members, representing a 'super' pro-apoptotic molecule for breast cancer cells, including CSCs. Second, the innovative gene therapy protocol developed by the authors using VISA-claudin4BikDD shows selective delivery of BikDD to cancer cells, with little or no effect on normal cells. Combining its potent pro-apoptotic activity with its selectivity for breast cancer cells, VISA-claudin4-BikDD enhanced the efficacy of lapatinib and killed paclitaxel-resistant CSCs. Therefore, BikDD represents a potential remedy for drug resistance. The evidence justifies the authors' intention to rapidly move this novel strategy to the clinical research setting.

Despite the comprehensive approach taken by Lang and colleagues, there are some unanswered questions, which should be considered during BikDD's translational path to the clinic. While improved efficacy of lapatinib was observed with BikDD, complete tumor eradication was still not achieved. This could be related to several factors. First, additional survival pathways beyond the key Bcl-2 family members may operate in breast CSCs. Second, VISA-claudin4-BikDD may not be able to infect and induce the expression of BikDD in all the tumor cells, including CSCs. Third, tumor cell and CSC heterogeneity may affect the overall efficacy of this therapy. In spite of these limitations, the novelty of delivering BikDD to breast cancer cells and its preclinical efficacy suggest that BikDD gene therapy may represent a new paradigm in the treatment of breast cancer patients.

\section{Abbreviations}

CSC, cancer stem cell; EGFR, epidermal growth factor.

\section{Acknowledgements}

This work was supported in part by NCI grants P50 CA58183 (Breast Cancer SPORE), by the Breast Cancer Research Foundation (BCRF) and the Stand Up to Cancer Breast Cancer Program.

\section{Author details}

'Lester and Sue Smith Breast Center at Baylor College of Medicine, Houston, TX 77030, USA. ${ }^{2}$ Department of Clinical Sciences and Administration,
University of Houston, College of Pharmacy, Houston, TX 77030, USA. ${ }^{3}$ Dan L Duncan Cancer Center at Baylor College of Medicine, Houston, TX 77030, USA. ${ }^{4}$ Margaret M and Albert B Alkek Department of Medicine at Baylor College of Medicine, Houston, TX 77030, USA. 5 Department of Molecular and Cellular Biology at Baylor College of Medicine, Houston, TX 77030, USA.

Published: 23 May 2012

\section{References}

1. Li X, Lewis MT, Huang J, Gutierrez C, Osborne CK, Wu MF, Hilsenbeck SG, Pavlick A, Zhang X, Chamness GC, Wong H, Rosen J, Chang JC: Intrinsic resistance of tumorigenic breast cancer cells to chemotherapy. J Natl Cancer Inst 2008, 100:672-679.

2. Phillips TM, McBride WH, Pajonk F: The response of CD24(-/low)/CD44+ breast cancer-initiating cells to radiation. J Nat/ Cancer Inst 2006, 98:1777-1785

3. Park CY, Tseng D, Weissman IL: Cancer stem cell-directed therapies: recent data from the laboratory and clinic. Mol Ther 2009, 17:219-230.

4. Konopleva M, Contractor R, Tsao T, Samudio I, Ruvolo PP, Kitada S, Deng X, Zhai D, Shi YX, Sneed T, Verhaegen M, Soengas M, Ruvolo VR, McQueen T, Schober WD, Watt JC, Jiffar T, Ling X, Marini FC, Harris D, Dietrich M, Estrov Z, McCubrey J, May WS, Reed JC, Andreeff M: Mechanisms of apoptosis sensitivity and resistance to the $\mathrm{BH} 3$ mimetic $\mathrm{ABT}-737$ in acute myeloid leukemia. Cancer Cell 2006, 10:375-388.

5. Liu G, Yuan X, Zeng Z, Tunici P, Ng H, Abdulkadir IR, Lu L, Irvin D, Black KL, Yu $J S$ : Analysis of gene expression and chemoresistance of CD133+ cancer stem cells in glioblastoma. Mol Cancer 2006, 5:67.

6. Ellis PA, Smith IE, Detre S, Burton SA, Salter J, A'Hern R, Walsh G, Johnston SR, Dowsett M: Reduced apoptosis and proliferation and increased $\mathrm{Bcl}-2$ in residual breast cancer following preoperative chemotherapy. Breast Cancer Res Treat 1998, 48:107-116.

7. Tabuchi Y, Matsuoka J, Gunduz M, Imada T, Ono R, Ito M, Motoki T, Yamatsuji T, Shirakawa Y, Takaoka M, Haisa M, Tanaka N, Kurebayashi J, Jordan VC, Naomoto Y: Resistance to paclitaxel therapy is related with $\mathrm{Bcl}-2$ expression through an estrogen receptor mediated pathway in breast cancer. Int J Oncol 2009, 34:313-319.

8. Martin AP, Miller A, Emad L, Rahmani M, Walker T, Mitchell C, Hagan MP, Park MA, Yacoub A, Fisher PB, Grant S, Dent P: Lapatinib resistance in HCT116 cells is mediated by elevated MCL-1 expression and decreased BAK activation and not by ERBB receptor kinase mutation. Mol Pharmaco/ 2008, 74:807-822.

9. Xia W, Bacus S, Hegde P, Husain I, Strum J, Liu L, Paulazzo G, Lyass L, Trusk P, Hill J, Harris J, Spector NL: A model of acquired autoresistance to a potent ErbB2 tyrosine kinase inhibitor and a therapeutic strategy to prevent its onset in breast cancer. Proc Natl Acad Sci U S A 2006, 103:7795-7800.

10. Kruyt FA, Schuringa JJ: Apoptosis and cancer stem cells: Implications for apoptosis targeted therapy. Biochem Pharmaco/ 2010, 80:423-430.

11. Lang JY, Hsu JL, Meric-Bernstam F, Chang CJ, Wang Q, Bao Y, Yamaguchi H, Xie X, Woodward WA, Yu D, Hortobagyi GN, Hung MC: BikDD eliminates breast cancer initiating cells and synergizes with lapatinib for breast cancer treatment. Cancer Cell 2011, 20:341-356.

12. Gupta PB, Onder TT, Jiang G, Tao K, Kuperwasser C, Weinberg RA, Lander ES: Identification of selective inhibitors of cancer stem cells by highthroughput screening. Cell 2009, 138:645-659.

13. Grimm D, Wehland M, Pietsch J, Infanger M, Bauer J: Drugs interfering with apoptosis in breast cancer. Curr Pharm Des 2011, 17:272-283.

14. Yerbes R, Palacios C, Lopez-Rivas A: The therapeutic potential of TRAIL receptor signalling in cancer cells. Clin Trans/ Oncol 2011, 13:839-847.

15. Del Poeta G, Bruno A, Del Principe MI, Venditti A, Maurillo L, Buccisano F, Stasi R, Neri B, Luciano F, Siniscalchi A, de Fabritiis P, Amadori S: Deregulation of the mitochondrial apoptotic machinery and development of molecular targeted drugs in acute myeloid leukemia. Curr Cancer Drug Targets 2008, 8:207-222.

doi:10.1186/bcr3125

Cite this article as: Giuliano M, et al:: Die and let live: harnessing BikDD to combat breast cancer stem cells. Breast Cancer Research 2012, 14:310. 